\title{
Moléstia de Chagas e ecologia profunda: a "luta antivetorial" em questão
}

\author{
Chagas's disease and deep ecology: \\ the anti-vectorial fight in question
}

Rodrigo Siqueira-Batista ${ }^{1}$

Andréia Patrícia Gomes ${ }^{1}$

Giselle Rôças ${ }^{2}$

Rosângela Minardi Mitre Cotta ${ }^{1}$

Eduardo Cárdenas Nogueira Rubião ${ }^{3}$

Alcides Pissinatti ${ }^{3}$

\footnotetext{
${ }^{1}$ Universidade Federal de

Viçosa. Av. Peter Henry

Rolfs s/n ${ }^{\circ}$, Campus

Universitário. 36570-000

Viçosa MG.

rsiqueirabatista@

yahoo.com.br

${ }^{2}$ Instituto Federal de

Educação, Ciência e

Tecnologia do Rio de

Janeiro.

${ }^{3}$ Centro Universitário Serra dos Órgãos.
}

\begin{abstract}
The inter-relations between man and the environment are among the main themes currently debated by the Brazilian public health. On such horizon, the questions concerning Chagas's disease are found to remain-specially in the scope of the directed actions of control to the triatomine, the anti-vectorial fight -, though already a century since its first description by Carlos Chagas, a major epidemiological problem in Latin America. Based on these considerations the present article will seek to discuss the main ecological aspects related to the American trypanosomiasis, emphasizing the control of the vectorial transmission in the context of the deep ecology.

Key words Ecology, Chagas's disease, Trypanosoma cruzi, Ethics, Health
\end{abstract}

Resumo As inter-relações entre o homem e o meio ambiente estão entre os principais temas debatidos no contexto atual da saúde pública brasileira. Neste horizonte se inscrevem as questões atinentes à moléstia de Chagas - especialmente no âmbito das ações de controle dirigidas aos triatomíneos, a luta antivetorial -, a qual permanece, mesmo após quase um século de sua descrição por Carlos Chagas, como uma condição de grande impacto epidemiológico na América Latina. Com base nestas considerações, o presente artigo buscará discutir os principais aspectos ecológicos relacionados à tripanossomíase americana - em um esforço de delimitação da questão, muito mais do que proposição de respostas -, enfatizando-se o controle da transmissão vetorial, repensada em termos da ecologia profunda.

Palavras-chave Ecologia, Moléstia de Chagas, Trypanosoma cruzi, Ética, Saúde 


\section{Introdução}

A moléstia de Chagas (ou tripanossomíase americana) - enfermidade parasitária causada pelo protozoário flagelado Trypanosoma cruzi e transmitida por insetos da família Triatominae $e^{1,2}$ - é uma antropozoonose restrita à América, com significativo impacto sanitário e socioeconômico no continente. Estima-se que a enfermidade atinja em torno de seis milhões de pessoas nos países nos quais grassa, ceifando, anualmente, cerca de 50 mil vidas $^{3-5}$.

Boa parte da história de misérias e sofrimentos relacionados ao mal de Chagas pode ser associada à perpetuação da transmissão vetorial, responsável pela maior parte do contingente de infectados por T. cruzi do planeta. De fato, díspares investigações demonstraram a existência de uma correlação direta entre a densidade doméstica de triatomíneos infectados e o número de casos agudos, especialmente nas crianças ${ }^{6,7}$. Assim, as ações de controle desenvolvidas ao longo do século XX - especialmente aquelas dirigidas à redução da transmissão por vetores - têm permitido uma transição epidemiológica ao menos em determinadas regiões do continente, sendo observada forte tendência à queda do número de casos em alguns países - como Argentina, Brasil (país que recebeu, recentemente, o Certificado Internacional de Eliminação da Transmissão da Doença de Chagas pelo Triatoma infestans $)^{8}$, Chile, Uruguai e Venezuela -, ainda que outros - como Bolívia, Paraguai e Peru - permaneçam com prevalências consideráveis, provavelmente pela implementação recente dos seus programas de controle ${ }^{9-11}$.

A despeito das benesses relacionadas às ações dirigidas aos vetores, é ilusório pensar que tal perspectiva, marcadamente belicista (marcial) fala-se da luta antivetorial, ou seja, da necessidade de exterminar os triatomíneos que insistem em manter-se nas vivendas humanas - seja capaz, efetivamente, de controlar a enfermidade. De fato, (1) a resistência dos transmissores aos diferentes inseticidas empregados; (2) as dificuldades de manejo do peridomicílio; (3) as questões relacionadas aos ciclos selváticos do T. cruzi (p. ex., na Amazônia); e (4) o fato de a moléstia de Chagas poder ser considerada uma doença genuinamente ecológica ${ }^{9,12}$ impõem que sejam pensados novos referenciais para a abordagem do problema, quiçá em uma perspectiva de conservação das espécies envolvidas, minimizandose o desequilíbrio ambiental.
É precisamente neste âmbito que ganha relevância a abordagem da enfermidade nos referenciais da ecologia - do grego óikos = morada; lógos = discurso; ou seja, estudo sobre a casa termo criado pelo biólogo Ernst Haeckel em $1866^{13}$. De fato, seu significado originário - saber que investiga a inter-relação dos seres vivos em um dado espaço geográfico (sua casa), estando, assim, intimamente relacionada às questões ambientais - foi, ao longo do século XX, ampliado sobremaneira, ganhando status de importante referencial teórico para se pensar o mundo contemporâneo - em termos éticos, políticos, epistemológicos e econômicos -, como demarcado por Leonardo Boff: [a ecologia de] um discurso regional como subcapitulo da biologia passou a ser atualmente um discurso universal, quiçá o de maior força mobilizadora do futuro milênio ${ }^{13}$.

A ecologia, assim, tem sido empregada para debater diferentes aspectos relacionados à vida na Terra - destacando-se os pontos dirigidos à saúde - no âmbito de uma concepção de integração dos seres vivos, aspecto que vem sendo considerado na abordagem da epidemiologia e do controle da tripanossomíase americana há décadas. Entretanto, a apreciação dos distintos elementos atinentes à moléstia de Chagas - em decorrência das múltiplas possibilidades de interação dos diferentes partícipes dos processos de perpetuação da doença, ou seja, o Trypanosoma cruzi, os triatomíneos e os mamíferos (incluído o Homo sapiens sapiens) - vem sendo realizada de modo marcadamente antropocêntrico, caracterizável, de acordo com os pressupostos de Arne Naess, como uma "ecologia rasa", a qual concebe os seres humanos como situados acima ou fora da natureza, atribuindo apenas um valor instrumental, ou de "uso", a esta última ${ }^{14,15}$.

Com base nestas premissas, discutir a luta antivetorial, partindo-se de uma apreciação ecoepidemiológica da doença, nos termos do arcabouço conceitual da ecologia profunda, é o escopo do presente artigo.

\section{Partícipes da teia ecoepidemiológica}

A compreensão da complexa "teia ecoepidemiológica” da tripanossomíase pressupõe o entendimento dos principais aspectos biológicos e ecológicos dos triatomíneos e dos reservatórios, os quais permitem a formulação de explicações para a circulação e a perpetuação do Trypanosoma cru$z i$ (Figura 1). 


\section{Os vetores: triatomíneos}

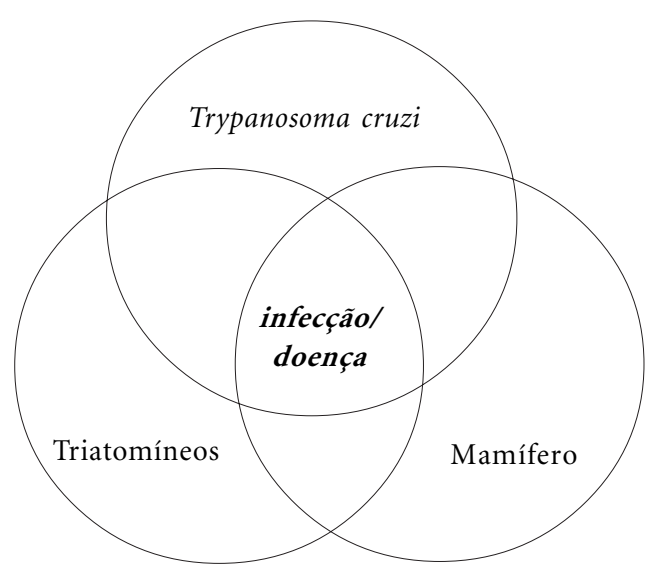

Figura 1. Interações dos participantes da teia ecoepidemiológica da moléstia de Chagas.
Os triatomíneos são insetos grandes, pertencentes à família Reduviidae e da subfamília Triatominae $^{16}$. São hematófagos obrigatórios, possuindo hábitos noturnos e longevidade de até dois anos ${ }^{17}$. O principal representante no Brasil é o Triatoma infestans - usualmente denominados como "barbeiro" -, a espécie mais importante na ecoepidemiologia da tripanossomíase americana no Brasil, tendo-se registrado sua ocorrência em mais de 720 municípios brasileiros ${ }^{3}$. Todavia, outras espécies possuem, também, significativa importância na ecologia da tripanossomíase americana, podendo-se citar Rhodnius prolixus, Panstrongylus megistus e Triatoma sordida ${ }^{18,19}$.

É amplamente reconhecido que o fenômeno mais importante na ecoepidemiologia da moléstia de Chagas é a domiciliação dos transmissores. Assim, em concordância com suas características bioecológicas e capacidade de domiciliação, os triatomíneos podem ser classificados em cinco grupos (Quadro 1) 20,21.

Quadro 1. Classificação dos triatomíneos vetores da moléstia de Chagas, como base em aspectos bioecológicos e capacidade de domiciliação.

\begin{tabular}{|c|c|c|}
\hline Grupos & Aspectos significativos & Principais espécies \\
\hline Grupo 1 & $\begin{array}{l}\text { Espécies com significativa adaptação } \\
\text { aos ecótopos artificiais; são raras ou } \\
\text { inexistentes nos ecótopos silvestres }\end{array}$ & $\begin{array}{l}\text { Triatoma infestans, Triatoma rubrofasciata e } \\
\text { Rhodnius prolixus (este último tem a particularidade } \\
\text { de poder ser encontrado tanto no ambiente silvestre, } \\
\text { especialmente em palmeiras, como no doméstico) }\end{array}$ \\
\hline Grupo 2 & $\begin{array}{l}\text { Espécies em movimento de } \\
\text { domiciliação; podem ser, com } \\
\text { frequência, encontradas em focos } \\
\text { silvestres }\end{array}$ & $\begin{array}{l}\text { Triatoma dimidiata, Triatoma sordida, Triatoma } \\
\text { maculata, Triatoma brasiliensis, Triatoma } \\
\text { pseudomaculata, Triatoma barberi, Triatoma } \\
\text { longipenis e Panstrongylus megistus (este último } \\
\text { mostra-se muito mais adaptado às habitações no norte } \\
\text { de Minas Gerais e Bahia, sendo predominantemente } \\
\text { peridomiciliar e silvestre ao sul de Minas Gerais e São } \\
\text { Paulo, e praticamente selvático em Santa Catarina) }\end{array}$ \\
\hline Grupo 3 & $\begin{array}{l}\text { Espécies prioritariamente silvestres; } \\
\text { eventualmente fazem incursões em } \\
\text { ecótopos artificiais, onde raramente } \\
\text { se encontram pequenas colônias }\end{array}$ & $\begin{array}{l}\text { Triatoma rubrovaria, Triatoma protracta, Triatoma } \\
\text { tibiamaculata, Triatoma vitticeps, Triatoma } \\
\text { matogrossensis, Rhodnius neglectus, Rhodnius nasutus, } \\
\text { Rhodnius pictipes, Rhodnius ecuadoriensis, Rhodnius } \\
\text { robustus e Rhodnius pallescens }\end{array}$ \\
\hline Grupo 4 & $\begin{array}{l}\text { Espécies essencialmente silvestres; } \\
\text { insetos adultos podem ser detectados, } \\
\text { de modo excepcional, em habitações } \\
\text { humanas, sem nunca colonizá-las }\end{array}$ & $\begin{array}{l}\text { Triatoma arthurneivai, Triatoma nitida, Triatoma } \\
\text { platensis, Panstrongylus geniculatus, Panstrongylus } \\
\text { lutzi, Panstrongylus diasi e outros }\end{array}$ \\
\hline Grupo 5 & Espécies exclusivamente silvestres & $\begin{array}{l}\text { Dipetalogaster maximus, Paratriatoma hirsuta, } \\
\text { Hermanlentia matsunoi, Mepraia spp, Psammolestes } \\
\text { spp, Cavernicola spp, Microtriatoma spp, Belminus } \\
\text { spp e outros }\end{array}$ \\
\hline
\end{tabular}


Outros protozoários, além do T. cruzi, podem infectar os triatomíneos - como Trypanosoma rangeli -, podendo haver risco de confusão diagnóstica nas áreas de ocorrência simultânea de ambos os agentes ${ }^{22,23}$.

\section{Os reservatórios: mamíferos}

Há inúmeras espécies de animais selváticos e domésticos que podem ser infectadas pelo T. cruzi, estando o Homo sapiens sapiens incluído neste último grupo ${ }^{24}$. Tais espécies são essenciais para a perpetuação da entidade mórbida ${ }^{25}$, apresentando processos infecciosos de variável gravidade - usualmente, mais benigna para os reservatórios silvestres do que para os domésticos ${ }^{20}$.

As aves, os répteis e os anfíbios são vertebrados naturalmente resistentes à infecção pelo hemoflagelado; todavia, esses animais - especialmente as aves - podem desempenhar significativo papel em alguns ecótopos, por servirem de fonte alimentar para os vetores ${ }^{3}$.

Quadro 2. Reservatórios silvestres do T. cruzi $^{26-29}$.

\begin{tabular}{|c|c|}
\hline Ordem & Espécies \\
\hline Carnivora & $\begin{array}{l}\text { Cerdocyon thous } \\
\text { Eira barbara } \\
\text { Nasua nasua }\end{array}$ \\
\hline Chiroptera & $\begin{array}{l}\text { Carollia perspicillata } \\
\text { Desmodus rotundus } \\
\text { Glossophaga soricina } \\
\text { Phyllostomus hastatus }\end{array}$ \\
\hline Edentata & Dasypus novemcintus \\
\hline Marsupialia & $\begin{array}{l}\text { Didelphis albiventris } \\
\text { Didelphis marsupialis }\end{array}$ \\
\hline Primates & $\begin{array}{l}\text { Aloutta spp } \\
\text { Ateles spp } \\
\text { Cacajao calvus } \\
\text { Callicebus personatus nigrifons } \\
\text { Callithrix spp } \\
\text { Cebus spp } \\
\text { Leontopithecus spp } \\
\text { Saguinus spp } \\
\text { Saimiri spp }\end{array}$ \\
\hline Rodentia & $\begin{array}{l}\text { Akodon spp } \\
\text { Coendu spp } \\
\text { Dasyprocta spp } \\
\text { Sciurus spp }\end{array}$ \\
\hline
\end{tabular}

\section{Reservatórios silvestres}

Os principais reservatórios do T. cruzi, no ciclo silvestre, são apresentados no Quadro $2^{26-29}$.

De modo similar ao descrito para os triatomíneos, deve-se destacar a possibilidade de infecção dos reservatórios selvagíneos por outros protozoários, o que eventualmente pode gerar equívoco. Pode-se citar, em relação aos primatas do Novo Mundo, a existência de pelo menos cinco espécies do gênero Trypanosoma capazes de infectá-los, incluindo Trypanosoma cruzi, Trypanosoma lambrechti, Trypanosoma rangeli, Trypanosoma minasense e Trypanosoma saimiri ${ }^{30,31}$. No que diz respeito às espécies do Velho Mundo (Macaca fascicularis, Macaca mulatta, Macaca cyclopis, Nycticebus concang, Hylobates pileatus), foi descrita uma forma pouco distinta de Trypanossoma cruzi que, para muitos autores, pode ser o Trypanossoma conorrhini, microrganismo encontrado em roedores na África ${ }^{32}$.

\section{Reservatórios domésticos}

Neste domínio, a espécie de maior importância é H. sapiens sapiens (homem), tendo em vista sua expectativa média de vida - cerca de 60-70 anos - e pela manutenção da parasitemia, ainda que baixa, por longos períodos. Este é infectado pelo protozoário, amiúde, no interior dos domicílios colonizados por triatomíneos ou por ocasião do desmatamento, em decorrência do desequilíbrio ecológico promovido (p. ex., em consequência à invasão dos acampamentos por vetores, os quais partem em busca de abrigo $)^{11,12}$.

Outros animais também atuam como reservatórios domésticos e peridomésticos do T. cru$z i$, tais como o cão (Canis familiaris) e o gato (Felis cattus) - vertebrados mais importantes, após o homem -, a cabra (Capra hyrcus), a ovelha (Ovis aries), o coelho (Oryctolagus cuniculis), a cobaia (Cavia porcellu) e o rato (Rattus novergicus $)^{3,33}$.

\section{Principais ecossistemas da moléstia de Chagas}

A infecção por T. cruzi se perpetua em díspares nichos ecológicos, organizando-se em significativa variedade de ambientes, com destaque para os ecótopos silvestres e domiciliares/peridomiciliares ${ }^{34}$.

\section{Ecótopos silvestres}

Acredita-se que, ancestralmente, o ambiente silvícola representava o único ecossistema do $T$. 
cruzi, como resultado da movimentação do protozoário entre diferentes reservatórios naturais especialmente marsupiais, roedores e edentados - e triatomíneos, em diferentes ecótopos ${ }^{25}$. Nesse domínio, propõe-se como significativa a transmissão por via oral do protista, havendo infecção dos reservatórios no momento da alimentação, como consequência da ingestão de triatomíneos ou de animais de menor porte, infectados ${ }^{35}$.

Os focos naturais da tripanossomíase - nos quais ocorreria o seu ciclo selvático - situam-se em distintos ambientes ${ }^{25,26,36}$ :

(1) Troncos das grandes árvores nas quais são abrigos de marsupiais (Didelphis spp., Marmosa spp., Caluromys), roedores, primatas (Calitriquídeos) e carnívoros, onde o triatomídeo Triatoma sordida alimenta-se do sangue desses mamíferos;

(2) Copas das grandes árvores - por exemplo, palmeiras -, com significativa participação de aves, marsupiais (Didelphis spp., Marmosa spp., Caluromys) e roedores - com registro do envolvimento de Panstrongylus megistus;

(3) Vegetação de cerrados e campos com envolvimento de marsupiais (Didelphis albiventris, Marmosa pusilla), carnívoros (Dusyceon griseus, Conepatus chinga, Galictis cuja, Herpailurus yaguaroundi), tatu (Tolypeutes mataco) e roedores (Microcavia australis e Pediolagua salinicola), com relato de Triatoma sordida e Triatoma infestans associados a esses animais selvagens;

(4) Ambientes subterrâneos (grutas, buracos no solo, sob pedras), destacando-se mamíferos - como Dasypus novemcinctus (tatu) - e espécies de triatomíneos (mormente Panstrongylus geniculatus);

(5) Rios e lagos, nos quais tomam parte Eira barbara (irara), mamífero com hábitos aquáticos, cuja infecção natural já foi descrita.

A despeito de se reconhecer alguns aspectos do comportamento selvagino da infecção por $T$. cruzi, muitos elementos permanecem por ser esclarecidos, reconhecendo-se que, em algumas regiões, são praticamente ignoradas as características ecológicas da moléstia de Chagas ${ }^{37}$.

\section{Ecótopos domiciliares e peridomiciliares}

A transmissão do T. cruzi ocorre, igualmente, em áreas peridomésticas e domésticas, as quais foram estabelecidas a partir de modificações dos hábitos humanos e animais. Ato contínuo à invasão desses ecótopos silvestres e construção de habitações que se tornaram propícias à domiciliação do vetor, o $H$. sapiens sapiens entrou em contato com o T. cruzi, havendo, assim, o surgimento da enfermidade humana ${ }^{38}$.

Não são muitas as espécies domiciliadas em torno de oito - entre as mais de 120 conhecidas. Ainda assim, há marcante coincidência entre a endemicidade da tripanossomíase americana e o grau de domiciliação dos triatomíneos. Nas regiões em que a domiciliação é significativa - como, por exemplo, em áreas da Bolívia e do Equador $^{3}$-, os vetores vivem preferencialmente em casas de "pau-a-pique", as quais possuem paredes com frestas utilizadas pelos artrópodos como esconderijo. São também encontrados, peridomiciliarmente, em galinheiros, chiqueiros, estábulos e casas de madeira. São insetos noturnos, saindo tanto o macho quanto a fêmea e ninfas, à noite, para o repasto sanguíneo. A despeito dessas conjecturas, recentes investigações realizadas a partir da caracterização de zimodemas silvestres e domiciliares demonstraram a circulação em condições selváticas dos zimodemas 1 e 3 , enquanto no ciclo doméstico detectou-se o zimodema 2, o que se torna um problema para a explicação desta continuidade entre os $\operatorname{ciclos}^{39,40}$.

\section{A questão ambiental: ecologia e saúde}

A questão ambiental - a qual tem como cerne os debates acerca da relação homem/ambiente emerge ao longo do século XX com a existência de duas correntes de pensamento - preservacionistas e conservacionistas -, as quais perpetuam os debates até a atualidade ${ }^{41,42}$. Os preservacionistas defendem que as belezas naturais devem permanecer intactas, não sendo alteradas por ações antrópicas, entendendo-se por preservação a proteção a longo prazo das espécies, habitats e ecossistemas. Já os conservacionistas percebem a natureza de forma mais sustentável, apresentando uma série de dados que indicam que o ambiente pode ser compartilhado pelo homem tendo em vista as suas necessidades, desde que se incorporem, às ações, os princípios do desenvolvimento sustentável. Propõem, de fato, o gerenciamento inteligente das terras e a utilização criteriosa dos recursos naturais de modo a gerar fontes de riqueza articulação entre economia e ecologia, ou seja, a utilização racional dos recursos naturais renováveis e não renováveis, objetivando produzir o maior benefício sustentado para as gerações atuais, mantendo suas potencialidades para satisfazer as necessidades das gerações futuras.

Seguindo a corrente conservacionista e como uma tentativa de consolidar as ações governa- 
mentais em prol do ambiente e dos direitos humanos, foi assinada a Agenda 21 na Conferência Rio- $92^{42}$. Nesse documento, foram traçadas as diretrizes sobre a forma segundo a qual deveriam ser conduzidas as discussões acerca das questões ambientais, resgatando a noção de desenvolvimento sustentável, proposta inicialmente no Relatório Brundtland, em 1987. Dentre os princípios norteadores do desenvolvimento sustentável, importa destacar duas de suas concepções básicas:

(1) atendimento às necessidades do presente sem comprometimento da possibilidade de as gerações futuras atenderem às suas próprias necessidades;

(2) requisição da participação dos cidadãos nos processos de tomada de decisão, na solução de problemas de ordem social, gerando um sistema econômico que seja confiável e constante.

Compondo a discussão político-econômica da questão ambiental, o mundo científico, representado pela Ecologia, vem apresentando uma série de indícios ${ }^{43,44}$, indicando que as atuações locais podem ter - e têm - resultados globais, interferindo assim nos padrões climáticos e ecossistêmicos do planeta Terra. Os desequílibrios ecológicos geram problemas de toda sorte, dentre os quais a propagação de espécies animais que são causadoras de moléstias humanas.

Particularmente no caso da moléstia de Chagas, percebe-se que o desmatamento da Floresta Amazônica está reduzindo habitats onde os predadores naturais do vetor vivem, havendo, assim, uma ausência do predador e o consequente aumento da população de triatomíneos. Ademais, há a possibilidade da geração de novos ambientes, nos quais o inseto pode ser ecologicamente mais eficiente. Nestes termos, reconhece-se que $a$ presença do barbeiro nas cidades está diretamente ligada a uma mudança de hábito do inseto, causada pelo aumento do desmatamento das florestas. [...] Se o contágio foi através das picadas, é a aproximação do barbeiro com as casas que preocupa, já que ele vive mais nas matas ${ }^{45}$.

Estudos sanitários e biogeográficos indicam que os triatomíneos possuem padrões de distribuição decorrentes da história natural desses protozoários ${ }^{46}$. Para tal entendimento, faz-se necessário que a discussão do tema esteja baseada em uma visão mais holística do ambiente reconhecendo-se sua totalidade e sua interdependência -, já que pode ser observado em distintos momentos que a moléstia de Chagas, tal qual outras doenças transmissíveis, é capaz de evoluir e se adaptar a novas condições ecológicas. Dessa forma, nos debates que envolvem temáticas relacionadas às questões ambientais e à saúde pública, torna-se imprescindível a discussão de forma mais abrangente - estabelecendo como ponto de referência o ecossistema -, revendo-se a tradicional perspectiva antropocêntrica.

\section{Luta antivetorial: uma crítica ecológica (profunda)}

A tripanossomíase americana é considerada, tradicionalmente, uma moléstia intimamente relacionada à miséria e à desigualdade social, tal qual denunciado por Carlos Chagas ainda no alvorecer do século passado ${ }^{47}$. Em grande medida, as profundas iniquidades sociossanitárias presentes na sociedade brasileira - quer entre regiões, quer entre pessoas de uma mesma região ou município - são determinadas sócio-historicamente $^{48}$. De fato, as condições de subdesenvolvimento dos países latino-americanos, capazes de impor a parcela significativa dos homens e mulheres dessas nações as mais espúrias condições de vida, estiveram entre os principais determinantes da grave situação ecoepidemiológica pela qual a doença se "arrastou" ao longo de seus cem anos de reconhecimento.

Tais foram os pressupostos que facultaram a compreensão, por parte da comunidade científica e da classe política mundiais, de que a guerra contra o mal de Chagas deveria ser uma batalha sem trégua pela melhoria das condições de vida das populações atingidas, mas, igualmente, contra os triatomíneos - inimigos que precisariam ser vencidos... -, sob pena de mais pessoas engrossarem os filões de vítimas da doença. Para isto, tem sido amplamente empregada, desde a década de 1940, a aplicação de inseticidas nos domicílios e peridomicílios, os quais atuam a partir de sua ação residual no sistema nervoso dos reduvídeos, especialmente nas ninfas (não atingem diretamente os ovos) ${ }^{49}$. Os produtos já utilizados incluem o BHC (hexaclorociclohexano), o Dieldrin, o Propoxur, o Malathion, os carbamatos - todos estes proibidos e proscritos em vários países - e os piretroides de síntese - principais produtos empregados atualmente -, tais como alfa-cipermetrina, beta-ciflutrina, ciflutrina, cipermetrina, deltametrina e lambda-cialotrina ${ }^{50}$. Este conjunto de ações - a luta antivetorial - foi um dos responsáveis pela substantiva redução do número de casos nos vários países da América (Gráfico 1) ${ }^{3}$.

Ainda assim, a luta antivetorial está longe de ser a melhor medida para que pessoas deixem de 
ser infectadas pelo T. cruzi, especialmente ao se reconhecer que o mal de Chagas não é apenas uma condição mórbida originada da pobreza e da desigualdade, mas sim uma enfermidade que possui tônicas raízes no desequilíbrio ambiental, ou seja: a moléstia de Chagas é, antes de tudo, uma doença ecológica. Algumas observações que ratificam tal posicionamento incluem:

(1) a crescente resistência dos triatomíneos aos inseticidas - como deltametrina, cipermetrina, beta-ciflutrina e lambda-cialotrina -, descrita em diferentes regiões da América Latina ${ }^{51,52}$, o que, na metáfora belicista vigente, representaria uma genuína reorganização das defesas triatomínicas;

(2) o grande problema representado pelo controle no peridomicílio, área na qual o tradicional uso de inseticidas tem pouca eficácia, pelo fato de a ação residual ser expressivamente inferior à descrita no domicílio; acrescente-se a isto o impacto ambiental e individual em termos de saúde humana e animal, em decorrência do uso desses venenos ${ }^{49}$;

(3) a ocupação de áreas consideradas livres do T. infestans pelo Triatoma braziliensis em regiões do Brasil - por exemplo, na área compre-

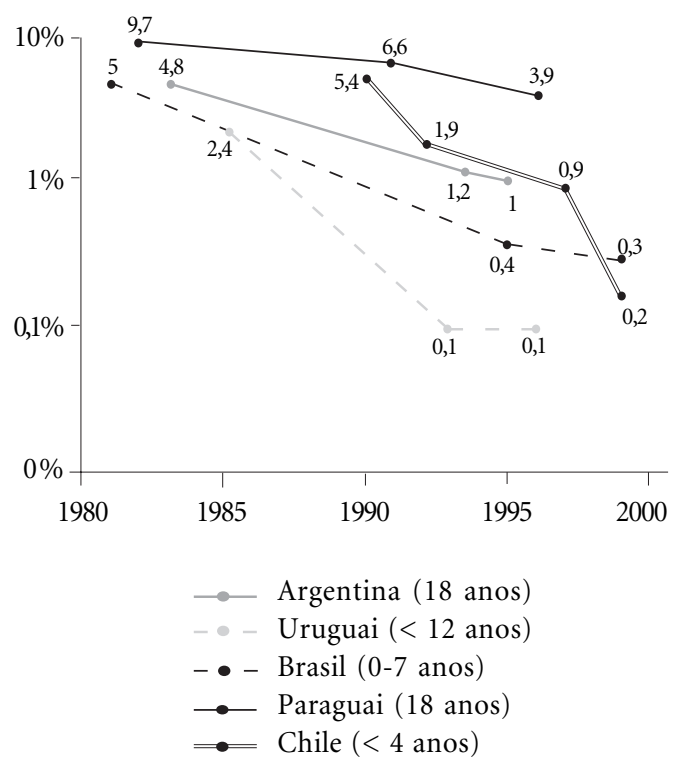

Gráfico 1. Tendência na redução da incidência de infecção por Trypanosoma cruzi nos países da América Latina. Fonte: WHO/OMS. World Health Organization. Control of Chagas disease. Second report of the WHO Expert Committee. Technical Report Series Geneva, 2002. endida pelas divisas dos estados de Tocantins, Piauí e Bahia -, bem como o ressurgimento do T. infestans na Argentina;

(4) as questões relacionadas aos ciclos selváticos da tripanossomíase americana, como no caso da Amazônia, localidade que vem sendo investigada, mais amiúde, nas últimas décadas, em relação às características da infecção pelo T. cru$z i$, cabendo destaque à baixa morbidade do processo infeccioso e à ocorrência de surtos de aquisição do protozoário por via oral; nesta região, tem-se descrito a ocorrência de inúmeros animais que participam como vetores e reservatórios (Quadro 3). Neste contexto, uma questão se impõe com força: seria plausível a realização de uma luta antivetorial - por exemplo, controle químico ou biológico de triatomíneos silvestres - nesses ecótopos naturais?

Com efeito, torna-se premente a revisão da forma segundo a qual se encaram as relações entre os diferentes elementos da teia ecoepidemiológica da tripanossomíase americana, podendo-se utilizar como referencial para pensar/agir a Ecologia Profunda, proposta em 1973 pelo filósofo norueguês Arne Naess ${ }^{14}$ - incluído na tradição de pensamento ecológico-filosófico de Henry Thoreau e de Aldo Leopold -, como alternativa ao modelo hegemônico de vigilância epidemiológica e controle das doenças (Quadro 4$)^{53}$. Neste domínio, para Capra: a ecologia profunda não separa seres humanos - ou qualquer outra coisa - do meio ambiente natural. Ela vê o mundo não como uma coleção de objetos isolados, mas como uma rede de fenômenos que são fundamentalmente interconectados e são interdependentes. A ecologia profunda reconhece o valor intrínseco de todos os seres vivos e concebe os seres humanos apenas como um fio particular na teia da vida ${ }^{15}$.

Em última análise, a ecologia profunda convida a que sejam pensadas e construídas novas relações entre os seres que habitam o planeta, por entender que há genuína igualdade entre as espécies e que a natureza tem um valor intrínseco, e não apenas instrumental (Quadro 3).

Tais ideias impõem que sejam pensadas inovadoras modalidades para o controle da moléstia de Chagas, as quais necessariamente terão que abandonar a ideia de que os triatomíneos são os inimigos que necessitam ser vencidos e aniquilados. Esta é, em última análise, uma genuína questão moral - mais do que técnica e/ou política tornando premente a introdução, nesses debates, dos referenciais teóricos da ética e da bioéti$\mathrm{ca}^{54,55}$, quiçá como formulado por Albert Schweitzer: Uma ética que nos obrigue somente a preocu- 
par-nos com os homens e a sociedade não pode ter esta significação. Somente aquela que é universal e nos obriga a cuidar de todos os seres nos põe de verdade em contato com o Universo e a vontade nele manifestada ${ }^{56}$.

Quadro 3. Principais espécies envolvidas nos ecossistemas amazônicas da moléstia de Chagas.

\begin{tabular}{|c|}
\hline $\begin{array}{l}\text { Triatomíneos } \\
\text { Eratyrus mucronatus } \\
\text { Panstrongylus geniculatus } \\
\text { Microtriatoma trinidadensis } \\
\text { Panstrongylus lignarius } \\
\text { Panstrongylus rufotuberculatus } \\
\text { Rhodnius brethesi } \\
\text { Rhodnius neglectus } \\
\text { Rhodnius paraensis } \\
\text { Rhodnius pictipes } \\
\text { Rhodnius robustus }\end{array}$ \\
\hline $\begin{array}{l}\text { Mamíferos } \\
\text { Agouti paca } \\
\text { Caluromys spp } \\
\text { Carollia perspicillata } \\
\text { Choeroniscus minor } \\
\text { Coendou spp } \\
\text { Cyclopes didactylus } \\
\text { Dasyprocta spp } \\
\text { Dasypus novemcinctus } \\
\text { Didelphis marsupialis } \\
\text { Echimys chrysurus } \\
\text { Eira barbara } \\
\text { Glossophaga soricina } \\
\text { Homo sapiens sapiens } \\
\text { Loncophylla mordax } \\
\text { Marmosa cinerea } \\
\text { Metachirus nudicaudatus } \\
\text { Molossus ater } \\
\text { Molossus molossus } \\
\text { Monodelphis brevicaudata } \\
\text { Mycronycteris megalotis } \\
\text { Nasua nausa [cf.] } \\
\text { Nectomys squamipes } \\
\text { Noctilio leporinus } \\
\text { Oryzomys capito } \\
\text { Philander opossum } \\
\text { Phyllostomus elongatus } \\
\text { Phyllostomus hastatus } \\
\text { Proechimys cayannensis } \\
\text { Rattus rattus } \\
\text { Saccopteryx bilineata } \\
\text { Saguinus midas niger } \\
\text { Saimiri sciureus } \\
\text { Sciurus spp } \\
\text { Tamandua tetradactyla }\end{array}$ \\
\hline
\end{tabular}

Fonte: Siqueira-Batista et al. ${ }^{12}$.
Como agir neste contexto? Eis a indagação ética por excelência. Pensar alternativas para o controle da moléstia de Chagas, no âmago das concepções da ecologia profunda, pode apontar saídas para os anos vindouros. Nestes termos, a orientação de investigações científicas para o desenvolvimento de modalidades vanguarda para a profilaxia e o controle - por exemplo, projetos para investigação (1) do impacto do desmatamento; (2) das ações em educação ambiental; e (3) de tecnologia em engenharia, de modo a tornar as moradias e o peridomicílio menos habitáveis pelos triatomíneos - seria uma medida bastante desejável. Evidentemente, não se trata de oferecer respostas - o que, no melhor espírito filosófico, foge do escopo do presente ensaio, muito mais dirigido à apresentação do problema -, mas apenas de se propor "linhas de força" para se pensar a cosmovisão - beligerante - na qual se inscreve a ideia de luta antivetorial.

Quadro 4. Comparação entre a visão de mundo hegemônica e a ecologia profunda.

\begin{tabular}{|l|l|}
\hline \multicolumn{1}{|c|}{$\begin{array}{c}\text { Visão de mundo } \\
\text { hegemônica }\end{array}$} & \multicolumn{1}{|c|}{ Ecologia profunda } \\
\hline Domínio da Natureza & $\begin{array}{l}\text { Harmonia com a } \\
\text { Natureza }\end{array}$ \\
\hline $\begin{array}{l}\text { Ambiente natural } \\
\text { como recurso para os } \\
\text { seres humanos }\end{array}$ & $\begin{array}{l}\text { Toda a Natureza tem } \\
\text { valor intrínseco }\end{array}$ \\
\hline $\begin{array}{l}\text { Seres humanos são } \\
\text { superiores aos demais } \\
\text { seres vivos }\end{array}$ & $\begin{array}{l}\text { Igualdade entre } \\
\text { as diferentes espécies }\end{array}$ \\
\hline $\begin{array}{l}\text { Crescimento } \\
\text { econômico e } \\
\text { material como base para } \\
\text { o crescimento humano }\end{array}$ & $\begin{array}{l}\text { Objetivos materiais a } \\
\text { serviço de objetivos } \\
\text { autorrealização }\end{array}$ \\
\hline $\begin{array}{l}\text { Crença em amplas } \\
\text { reservas de recursos }\end{array}$ & $\begin{array}{l}\text { Planeta tem recursos } \\
\text { limitados }\end{array}$ \\
\hline $\begin{array}{l}\text { Progresso e soluções } \\
\text { baseados em alta } \\
\text { tecnologia }\end{array}$ & $\begin{array}{l}\text { Tecnologia apropriada } \\
\text { e ciência não dominante }\end{array}$ \\
\hline $\begin{array}{l}\text { Consumismo } \\
\text { acomunidade } \\
\text { nacional centralizada }\end{array}$ & $\begin{array}{l}\text { Biorregiões } \\
\text { e reconhecimento } \\
\text { de tradiços das minorias }\end{array}$ \\
\hline necessário e reciclando \\
\hline
\end{tabular}

Fonte: Goldim ${ }^{53}$. 


\section{Ponderações finais}

Transformar, de forma premente e radical, os debates sobre a epidemiologia, a ecologia, a prevenção e o controle da infecção chagásica, permitindo que a coletividade possa se tornar, finalmente, responsável pelo seu entorno - vertebrados, T. cruzi e triatomíneos -, quiçá como esperado por Albert Schweitzer, é tarefa urgente e de responsabilidade de todos os agentes morais, racionais e autônomos, incluindo-se os diferentes setores sociais - sociedade civil organizada, profissionais de saúde, gestores, comunidade científica, classe política, membros dos Conselhos de Saúde nos três níveis de governo. O que está em jogo é um expressivo número de vidas - basta observar, conforme demonstrado, o quantitativo de espécies partícipes nos ecossistemas envolvidos na moléstia de Chagas -, as quais não podem ser tratadas meramente como meio para manutenção da saúde humana, estabelecendose, quiçá, uma ampliação do imperativo kantiano: em vez de "Trata a humanidade, na tua pessoa ou de outros, sempre como um fim e nunca somente como um mero meio", reescrever: "Trata todos os seres, sempre como um fim e nunca somente como um mero meio"s7.

Com base nessas considerações, deve-se trabalhar para que as novas - ou renovadas - discussões sobre a ecopidemiologia da tripanossomosíase americana não perca de vista os elementos do debate moral contemporâneo - tais como a ética do cuidado ${ }^{58}$, a bioética da proteção ${ }^{59} \mathrm{e}$ a bioética para todos os seres (ou bioética quântica $)^{60}-$, os quais têm enfatizado, em grande medida, a necessidade de se reconsiderar a compaixão (laica) como um dos pontos fundamentais para a construção de um mundo mais justo, equânime e fraterno.

\section{Colaboradores}

R Siqueira-Batista, AP Gomes, G Rôças, RMM Cotta, ECN Rubião e A Pissinatti participaram igualmente de todas as etapas da elaboração do artigo. 


\section{Referências}

1. Siqueira-Batista R, Geller M, Martins AV, Bastos OMP. Trypanosoma cruzi. In: Siqueira-Batista R, Corrêa AD, Gomes AP, Geller M. Moléstia de Chagas. 2a ed. Rio de Janeiro: Rubio; 2007.

2. Almeida PS, Ceretti Júnior W, Obara MT, Santos HR, Barata JMS, Faccenda O. Levantamento da fauna de Triatominae (Hemiptera: Reduviidae) em ambiente domiciliar e infecção natural por Trypanosomatidae no estado de Mato Grosso do Sul. Rev Soc Bras Med Trop 2008; 41:374-380.

3. World Health Organization (WHO/OMS). Control of Chagas disease: second report of the WHO Expert Committee. Geneva: WHO; 2002. (Technical Report Series Geneva).

4. Dias JCP. Epidemiological surveillance of Chagas disease. Cad Saude Publica 2000; 16(Suppl.2):43-59.

5. Dias JCP. Controle da doença de Chagas no Brasil. In: Silveira AC. El control de la enfermedad de Chagas en los países del Cono Sur de América. Uberaba (MG): Faculdade de Medicina do Triângulo Mineiro; 2002.

6. Montoya R, Dias JCP, Coura JR. Chagas disease in a community in Southeast Brazil. I. A serologic follow-up study on a vector controlled area. Rev Inst Med Trop Sao Paulo 2003; 45(5):269-274.

7. Dias JCP. Epidemiologia. In: Brener Z, Andrade ZA, Barral-Netto M, organizadores. Trypanosoma cruzi e doença de Chagas. Rio de Janeiro: Guanabara Koogan; 2000.

8. Organização Pan-Americana da Saúde (Opas). Enfermedad de Chagas (tripanosomiasis americana). [acessado 2006 jul 15]. Disponível em: http:// www.paho.org/spanish/ad/dpc/cd/chagas.htm

9. Carlier Y, Dias JCP, Luquetti AO, Hotenbeyrie M, Torrico F, Truyens C. Trypanosomiase américaine ou maladie de Chagas. Enciclop Méd-Chirurgicale 2002; 8:505-520.

10. Dias JCP. Presente e futuro do controle dos triatomíneos vetores de Chagas no Brasil. Rev Soc Bras Med Trop 1989; 22(Supl.II):5-10.

11. Dias JCP. Situación actual de la enfermedad de Chagas. In: Madorry C, Camera ML, editores. Actualizaciones en la enfermedad de Chagas. Buenos Aires: Congresso Nacional de Medicina; 1993.

12. Siqueira-Batista R, Gomes AP, Rubião ECN, Gonçalves MLC. Infecção por Trypanosoma cruzi na Amazônia. In: Siqueira-Batista R, Corrêa AD, Gomes AP, Geller M. Moléstia de Chagas. 2a ed. Rio de Janeiro: Rubio; 2007.

13. Boff L. Ética da vida. Rio de Janeiro: Sextante; 2005.

14. Naess A, Rothenberg D. Ecology, community and lifestyle: outline of an ecosophy. Cambridge: Cambridge University Press; 1990.

15. Capra F. A teia da vida: uma nova compreensão científica dos sistemas vivos. $6^{a}$ ed. São Paulo: Cultrix; 2001.

16. Lent H, Wygodzinsky P. Revision of the Triatominae (Hemiptera, Reduviidae), and their significance as vectors of Chagas' disease. Bull Am Mus Nat Hist 1979; 163:123-520.

17. Vahia-Loureiro AM, Siqueira-Batista R, Corrêa AD, Ramos Júnior AN, Huggins DW, Quintas LEM. Biologia dos triatomíneos. Arq Brasil Med 1996; 70:623625 .
18. Abad-Franch F, Palomeque FS, Aguilar HM, Miles MA. Field ecology of sylvatic Rhodnius populations (Heteroptera, Triatominae): risk factors for palm tree infestation in Western Ecuador. Trop Med Int Health 2005; 10(12):1256-1258.

19. Brasil. Ministério da Saúde. Secretaria de Vigilância em Saúde. Consenso Brasileiro de Doença de Chagas. Rev Soc Bras Med Trop 2005; 38(Supl.III):1-29.

20. Ferreira MS, Lopes ER, Chapadeiro E, Dias JCP, Ostermayer AL. Doença de Chagas. In: Veronesi R. Tratado de infectologia. $3^{a}$ edição. Rio de Janeiro: Atheneu; 2005.

21. Silveira AC. Current status of vector transmission control of Chagas' disease in the Americas. In: Carcavallo R, Gallindez Girón I, Jurberg J, Lent H, editors. Atlas dos vectores da Doença de Chagas nas Américas. v. 1 e 2. Rio de Janeiro: Fiocruz; 1999.

22. Azambuja P, Garcia E. Trypanosoma rangeli interactions within the vector Rhodnius prolixus - a mini review. Mem Inst Oswaldo Cruz 2005; 100(5):567572.

23. Siqueira-Batista R, Vahia-Loureiro AM, Quintas LEM, Corrêa AD, Huggins DW. Trypanosoma rangeli. Rev Bras Med 1998; 55:423-426.

24. Dias JCP. Epidemiology of Chagas' disease. In: Wendel S, Brener Z, Camargo ME, Rassi A, editors. Chagas Disease (American Trypanosomiasis): it's impact on transfusional and clinical medicine. São Paulo: ISBT Brazil' 92; 1992.

25. Corrêa AD, Miranda Filho N, Siqueira-Batista R, Huggins DW. Papel dos reservatórios na epidemiologia da moléstia de Chagas. Rev Bras Med 1998; 55:414-420

26. Siqueira-Batista R, Rubião ECN, Cotta RMM, Pissinatti A, Soranz DR, Pinto LFS. Epidemiologia e ecologia. In: Siqueira-Batista R, Corrêa AD, Gomes AP, Geller M, organizadores. Moléstia de Chagas. 2a ed. Rio de Janeiro: Rubio; 2007.

27. Deane LM. Infecção natural do sagui Callithrix jacchus por tripanosoma do tipo cruzi. Rev Inst Med Trop 1962; 4:225-229.

28. Lisboa CV, Mangia RH, Rubião ECN, Lima NRC, Xavier SCC, Pissinatti A, Ferreira LF, Fernandes O, Jansen AM. Trypanosoma cruzi transmission in a captive unit, Rio de Janeiro, Brazil. Acta Tropica 2004; 90(1):97-106.

29. Moreira GV, Serra Peixoto CM, Ziccardi M, Oliveira RL, Castro MG, Dionísio DF, Pissinatti A. Prevalência de Trypanosoma cruzi, Trypanosoma minasense e de anticorpos contra arbovírus em primates não humanos (Callithrichidae) mantidos em cativeiro. Rev Bras Med Vet 2000; 22(6):252-254.

30. Ziccardi M, Lourenço-de-Oliveira R, Lainson R, Brigido MC, Muniz JA. Trypanosomes of non-human primates from the National Centre of Primates, Ananindeua, State of Para, Brazil. Mem Inst Oswaldo Cruz 2000; 95(2):157-159.

31. Ziccardi M, de Oliveira RL, Alves MC, da Cruz MF. Trypanosoma saimirii Rodhain, a junior synonym of Trypanosoma rangeli Tejera. J Parasitol 2005; 91(3):653-656. 
32. Cross JH, Hsu MYK, Hung CK. Studies in trypanosomes in the Taiwan monkey. The Southeast Asian Journ of Tropical Medicine and Public Health 1983; 14:536-542.

33. Fernandes AJ, Diotaiuti L, Dias JCP, Romanha AJ, Chiari E. Inter-relações entre os ciclos de transmissão do Trypanosoma cruzi no município de Bambuí, Minas Gerais, Brasil. Cad Saude Publica 1994; 10(4):473-480.

34. Nakamura A, Rubião ECN, Siqueira-Batista R. Principais espécies de triatomíneos implicadas na transmissão da moléstia de Chagas. In: Siqueira-Batista R, Corrêa AD, Gomes AP, Geller M. Moléstia de Chagas. 2a ed. Rio de Janeiro: Rubio; 2007.

35. Dias JCP. Notas sobre o Trypanosoma cruzi e suas características bioecológicas, como agente de enfermidades transmitidas por alimentos. Rev Soc Bras Med Trop 2006; 39(4):370-375.

36. Schweigmann NJ. Aspectos ecológicos de uma población santiaguena de la comadreja overa (Didelphi albiventris) en relación con la transmisión del Trypsanosoma cruzi [tese]. Buenos Aires: Universidad de Buenos Aires; 1994.

37. Noireau F, Carbajal-de-la-Fuente AL, Lopes CM Diotaiuti L. Some considerations about the ecology of Triatominae. An Acad Bras Ciênc 2005; 77(3):431436.

38. Dias JCP, Macedo VO. Doença de Chagas. In: Coura JR, organizador. Dinâmica das doenças infecciosas e parasitárias. Rio de Janeiro: Guanabara Koogan; 2005.

39. Lisboa CV, Mangia RH, Pissinatti A, Rubião E, Ivo A, Fernandes O, Jansen AM. The transmission of Trypanosoma cruzi among neotropical primates from Brazil. In: Resumos do Simpósio Internacional sobre avanços do conhecimento da Doença de Chagas 90 anos após a sua descoberta. Rio de Janeiro; 1999.

40. Santos SS, Carrara N, Junqueira ACV, Cupolillo E, Fernandes O. Molecular analysis of the genetic variability of Brazilian Trypanosoma cruzi isolates. In: Resumos do Simpósio Internacional sobre Avanços do Conhecimento da Doença de Chagas 90 Anos após a sua Descoberta. Rio de Janeiro; 1999.

41. Philippi Jr. A. Saneamento, saúde e ambiente: fundamentos para um desenvolvimento sustentável. São Paulo: Manole; 2005.

42. Malheiros TF, Philippi Jr. A, Coutinho SMV. Agenda 21 nacional e indicadores de desenvolvimento sustentável: contexto brasileiro. Saude Soc 2008; 17(1):7-20.

43. Odum EP, Barrett GW. Fundamentos de ecologia. 5a ed. São Paulo: Thomson Learning; 2007.

44. Carvalho, ICM. Educação ambiental: a formação do sujeito ecológico. 2a ed. São Paulo: Cortez; 2006.

45. Santos EO. Especialistas alertam para risco da Doença de Chagas. O Liberal 2006; 29 jul. [acessado 2007 jun 27]. Disponível em: http://iah.iec.pa.gov.br/ iah/fulltext/bvssite/noticias/29jul2006oliberal.pdf

46. Silva LJ. Comentário: releitura e homenagem à biogeografia, origem e distribuição da domiciliação de triatomíneos no Brasil. Rev Saude Publica 2006; 40(6):999-1000.
47. Chagas C. Segunda conferência realizada na Academia Nacional de Medicina, em agosto de 1911. In: Prata A, organizador. Carlos Chagas. Brasília: Ed. Universidade de Brasília; 1981. (Coletânea de Trabalhos Científicos).

48. Siqueira-Batista R. Miséria. 3a ed. Rio de Janeiro: KroArt; 2003.

49. Ramos Júnior AN, Pontes RJS, Mello MGS, Heukelbach J. Profilaxia e controle. In: Siqueira-Batista R, Corrêa AD, Gomes AP, Geller M, organizadores. Moléstia de Chagas. 2a ed. Rio de Janeiro: Rubio; 2007.

50. Ramsey JM, Schofield CJ. Control of Chagas disease vectors. Salud Publica Mex 2003; 45:123-128.

51. Gentile AG, Sartini JL, Campo MC, Sanchez JF. Efficacy of Fipronil in the control of the peridomiciliary cycle of Triatoma infestans in an area resistant to Deltamethrin. Cad Saude Publica 2004; 20:1240-1248.

52. Picollo MI, Vassena C, Santo OP, Barrios S, Zaidemberg M, Zerba E. High resistance to pyrethroid insecticides associated with ineffective field treatments in Triatoma infestans (Hemiptera: Reduviidae) from Northern Argentina. J Med Entomol 2005; 42:637-642

53. Goldim JR. Ecologia profunda. [acessado 2005 set 8]. Disponível em: http://www.ufrgs.br/bioetica/ ecoprof.htm

54. Potter VR. Bioethics, science of survival. Biology and Medicine 1970; 14:153-173.

55. Potter VR. Bioethics: bridge of the future. Englewood: Prentice-Hall; 1971.

56. Schweitzer A. Decadência e regeneração da cultura. São Paulo: Melhoramentos; 1964

57. Siqueira-Batista R. Trata todos os seres sempre como um fim e nunca somente como um mero meio matizes conceituais da bioética quântica. In: III Jornada Científica da Baixada Fluminense; 2008. Nilópolis: Centro Federal de Educação Tecnológica de Química de Nilópolis; 2008.

58. Boff L. Saber cuidar: ética do humano - compaixão pela Terra. 8a ed. Petrópolis (RJ): Vozes; 2002.

59. Schramm FR. Bioética da proteção: justificativa e finalidades. Iatrós - Cadernos de Filosofia, Saúde e Cultura 2005; 1:121-130.

60. Siqueira-Batista R. Quantum bioethics: ethics for all beings. Cien Saude Colet 2008; 13(3):611-613.

Artigo apresentado em 03/07/2007

Aprovado em 07/04/2008

Versão final apresentada em 12/11/2008 